\title{
ESTRATIGRAFIA PRETERCIARIA PRELIMINAR DEL ESTADO DE CHIAPAS
}

Por Federico K. G. Múflertied.

Estratigráficamente la parte sureste del país es paco conocida, como sucede con axuella porción que.corresponde al Estado de Chiapas. Me limito a exponer. su estratigrafía prẹterciaria, porque el estudio del Terciario de Chiapas no se puede hacer en México, en vista de que existe poco material paleontológico de comparación en las colecciones de? Instituto de Geología y por la eSCasa literatura universal estratigráfica y paleontológica de nuestra biblioteca, ambos indispensables para un reconocimiento estratigráfico serio.

La estratigrafía preterciaria de Chiapas, debe basarse en primer lugar en fósiles característicos, sin los cuales no se puede hablar de estratigrafía, sino solamente de lineamientos generales.

El primero que inició la paleontología preterciaria de ese Estado, fué el señor profesor José G. Aguilera, porque.determinó algunos fósiles traídos por el señor ingeniero Próspero Goyz-ueta, miembro de la Comisión encargada de trazar los limites entre México y Guatemala (I. 1 ). Poco después de Goyzueta, Carlos Sapper colectó fósís en dicho Estado, que füeron clasificalos por K. von Zittel, E. Stolley y F. von Roemer $(9,10.11)$; y postriormente Boese (2) recogió fósiles y m-odi;ficó. en parte, lo dicho por Sapper. No siendo sino hasta el año de $\mathbf{1 9 2 5}$ cuando W. A: Verwiebe (12) col-ectó también algumos fósiles en Chiapas y Waibel (13) aparentemente no 108 encontro.

Hace diez años, el que esto. escribe, inicjó una investigación geológica en el Estado de Chiapas por indicación del 
señor ingeniero J. D. Villareilo. Hasta la fecha he podido hacer ocho viajes de estudio y he traído bastantes fósiles que, me permiten establecer una estratigrafia más rigUl"OSa que la existente hasta aihora.

Aguilera tuvo el honor de haber fundado la estratigrafía de Chiapas, porque los fósiles colectados por Goyzueta, fueron los primeros evaluados estratigráficamente e indican, según Aguilera, la existencia de la caliza carbonífera. Los fósiles recolectados por Sapper en Chiapas y en la vecina República de Guatemala, demuestran según K. von Zittel, E. Stolley y F. von Roemer la presencia de capas del Carbonjfero y del Cretácico. Por cuestión de superposición, Sapper' reconoció el Devónico (estratos de Santa Rosa) y capas precretácicas (estratos de Tơdos Santos), siendo el mi'smo Sapper quien reconoció como formación más antigua del Estado, el Azojco, al que correspondían en aquella época todas las rncas prepaleozoicas. Los términos locales se refieren a pueblos guatemaltecos, donde las respectivas capas de Chiapas afIaran de una manera perfecta. F. H. Girty (14) considera los fósiles recogidos por Sapper en las capas finales de los estratos de Santa Ros̀a, como del Pennsylvaniano inferior, o posiblemente Mississipiano superior y los de las calizas sobrepuestas como del Pennsylvaniano.

Boese distingue el Arcaico, el Devónico? (División de Santa Rosa), el Carbonífero, el Triásico? (División de Todos Santos), el Cretácico inferior yel Cretácico medio. (Caliza de Rudistas);

Falta tal vez, según Boese, el Cretácico superior. Lo que Boese aportó a la estratigrafía de Chiapas es entonces, poco diferente a lo que Sapper comunicó. Lo mismo se pu-ede de. cir de W. A. Ver Wiebe, quien reconoció el Areajco, Devónica, Carbonífero, Triásico (en parte Jurásico?, capas de Todos Santos), el Jurásico (capas de Tuxtla), y parte inferior del Cretácico o Comanche (capas de San Cristóbal).

Por último, Waibel no trae datos estratigráficos nuevos, porque cita las formaciones ya reoonocidas por Sapper y Baese. 
Mis exploraciones e investigaciones detalladas en Chiapas, tenían por objeto delinear las zonas petrolíferas. Encontrándome con una estratigrafía deficiente, traté de establecer una estratigrafía más rigurosa. Ya en las primeras excursiones tuve cierto éxito y sobre todo en las últimas- detalladas, que se hacían gracias al permiso del señor profesor Isaac Ochoterena, Director del Instiuto de Biología y a la recomendación del Gobernador actual de Chiapas. coronel Vietórico Grajales. Aprovechando esta oportunidad me complazco en dar las debidas gracias a mis guías, al pueblo chiapaneco en general, por su hospitalidad y ayuda en mis pesquisas paleontológicas e investigaciones estratigráficas, lo mismo que a los señores Federico Hommel y Herbert Zimmermann, dueño y administrador, respectivamente, de la finca "El Suspiro".

El resultado principal de mis estudios es la comprobación de la existencia de las siguientes formaciones preterciarias en Chiapas: Arcaico-Arquezoico, Paleozoico superior, Jurásico y Cretácico (3 a 8).

La formación más antigua es la del Arcaico-Arqueozoico, que se encuentra af,lorando en el suroeste del Estado. La mayor parte de la llamada Sierra Madre, se compone de roas del Arcaico-Arqueozoico que tambjén afloran en algunas porciones de la planicje costera del Pacífico. Son rocas intrusivas y metamorfizadas que no contienen fósiles y cuya edad estratigráfica es conocida, porque en la región de Motozintla quedan sobrepuestas por los estratos del Palezoico superior. La f.ormación subyacente debe ser más antigua, y mucho más, porque hay discordancia entre las dos formaciones. La edad prepaleozoica de estas rocas antiguas es muy proba:ble, porque la conocemos continuando con interrupcionos por todo el sur y poniente de México, assí como porque penetran a territorjo de los Estados Unidos del Norte, donde están cubiertas por capas paleozoicas y aún por sus capas basales: el Cámbrico. Por lo tanto, deben ser mís antiguas que el mismo Cámbrioo o prepaleozoicas, -eS decir, ArcaicoArqueozoico sin que se pueda precisar una edad más rigoro- 
sa, esto es, Arqueozoico y Arcaico o cierta parte de estas formaciones. En este sentido hay que tomar el término indicado Arcaico-Arqueozoico. Las antiguas rocas intrusivas y me. tamorfizadas de Chiapas, son de edad prepaleozoica, aunque tal rez, con excepeión de parte de ellas qua, pueden ser paste. riores y que fueron metamorfizadas o intrusionadas pastarior: mente, por lo que tienen un aspecto antiguo, pero una edal diferente, como se conoce en la parte sur del Istmo de Te. huantepec (Ch. L. Baker: Geological cross-section of Isthmus of Tehuantepec.-Pan. Am. Geo1., Vol. 53, p. 161, 1930) .

Solamente futuras investigaciones podrán demostrarnos si todas las rocas antIguas de Chiapas, son de edad prepaleozoica o parte de ellas son de edad algo más re ciente. Las rocas verdaderamente muy antiguas forman fuera: de la región de afloramiento, la base de todos los estratos y rocas posteriores en Chiapas.

Encima del complejo basal hay una serie de estratos de edad suprapaleozoica y mesozoica.

De ésta la porción más antigua pertenece al Paleozojco superior y aflora en la región al sur de Ohicomucelo. Sapper 'reconoció una parte inferior de areniscas (estratos de Santa Rosa) y otra superior de calizas; en las areniseas no hay fósiles con excepción de los bancos finales, pero en las calizas sí hay bastantes fósiles marinos. Existen 18 formas diferentes de foraminíferos, gasterópodos, braquiópodos, crinoides, amonitas, lamelibránquios, briozoarios y vermes, según los hallazgos de Sapper, Boese y del autor. Los fósi].es son de edad no solamente del Ca1rbonífero superior y tal vez final del Carboníf.ero inferior (según F. H. Girty), sino también en parte del Pérmico, como puedo demostrarlo, puesto que encontré amonitas mal conservadas del género Waagenoce. ras o de un género muy cercano a éste, que se conocen ax. clusivamente en el Pérmieo. Además, encontré dos especies de gasterópodos: Plenrotornaria cf. carinifera Girty y P.ne. glecta Girty, ambas conocidas en el Pérmico de Texas; los demás fósiles no son del Pérmico, sino del Carbonífero supe- 
rior. F. H. Girty, como ya indiqué, considera estos fósiles encontrados en las capas finales de la arenisca, como del PHmsylvaniano inferior o posiblemente del Mississippiano superior, y los de las calizas como del Pennsylvaniano. He podido encontrar la Fusulina cí. pseudo-prisca em. CoJani (Palaeofusulina prisca Deprat) que también indica una edad Carbonifera superior(Pennsylvaniano).

La serie suprapaleozoica en cuestión es por lo tanto, no del Devónico y Carbonífero, sino del Carbonífero (estratos de Santa Rosa y part.e inferior de las calizas) y del Pérmico (parte superior de las ealizas) que tienen en conjunto un pesor de muchos centenares de metros. En la actualidad no' es posible indicar rigurosamente làs zonas paleontológicas y !a sucesión de éstas. Me permito señala'r que en algunas otras regiones de México tampoco conocemos el Devónico y Carbonífero, sino el Paleozoico superior de facies marina en el norte, en los Estados de Sonora, Coahuila y Tamaulipas y en el sur, en el I'stmo de Tehuantepec; y de estas regiones Chiapas no forma una excepción como se creía anteriormente. Por esto, no nos sorprende que el Paleozoico su. perior de Chiapas tenga relaciones faunísticas con las respec. tivas capas del suroeste de los Estados Unidos de Norte América. Afloran las capas en Chiapas en el triángulo Motozintla, Chicomucelo, San José Montenegro, zona que continúa en "la vecina región de Guatemala y también hacia el N.E. donde están cubiertas de formaciones más recientes. Pudiera ser que haya otrosafloramientós del Palezoico superior entre Ohicomucelo y el Istmo de Tehuantepec, donde se conoce también el Paleozoico superior; pero estos terrenos, en partee, no están explorados toda:vía.

Una extensión mucho, mayor tienen los estratos del Mesozoico en Chiapas, que se encuentran principalmente al N.E. de la Sierra Madre, ocupando una enorme extensión en el Estado y entrando también a algunas porciones del N.E. de la Sierra Madre. La porción inferior de la serie Mesozoiea se compone esencialm.ente de areniscas y pizarras con restos 
vegetales, y la superior principalmente de calizas, así como también de areniscas y margas, con invertebrados marinos.

La porción inferior corresponde a los estratos de Todos Santos de Sapper, cuya parte superior ha sido considerada como Cretácico inferior por Ecese, porque está debajo del ,Cretáeico medio. La edad infracretácica es cierta, puesto que he podido encontrar fósiles del Neocomiano, no conocidos por Boese. Estos, son 49 especies de lamelibránquios, gasterópocios, braquiópodos, foraminíferos y restos vegetales. De los lamelibránquios y gasterópodos nueve especies son características del Neocomiano de los montes Jura de Suiza y Francra, y también de otras partes de Europa, Asia, Africa, Sud América y California:

Nerinea? Douvillei Choffat,

Nerinella? lobata d'Orb.,

Harpagodes aff. Desori P. y C.,

Natica? Pilleti, Chofat,

Natica aff. Ampullaria laevigata Desh.,

Venus Dupiniana d'Orb.,

Panopaea gurgitis Brogn.,

Protocardia peregrina d'Orb.,

Nucula Gabbi? 8tanton (parte superior de los estratos de Knoxville, California).

Dos especies más, la Natica? Dido Krumbeck y la Terebratula subsella Leym., más bien indican una edad suprajurásica, pero el género Ptychomya también es,tá presente y se encuentra exclusivamente en capas cretácicas. Las especies citadas, por consiguiente, indican la edad Neocomiana de las respectivas capas, bajo las cuales yace concordantemente el Jurásico. Su parle final consiste de unos 100 metros de pizarras, calizas, caliza con huecos, yeso y arenisca y pizarra de color rojo. Los fósiles que encontré están muy mal conservados, pero entre ellos reconocí una Nerinea con alguna probabilidad; Ostrea sp., fragmentos de lamelibránquios o braquiópođos, todos fósiles marinos. Más abajo quedan al. gunos centenares de metros de areniscas y areniscas conglo- 
meráticas, en parte de color rojo, pizarras y margas, con madera fósil y hojas.

La serie que yace concordantemente debajo del Neocomiano tiene entonces una subdivisión en das partes, conocida ya en el N.O. de Oaxaca y N. de Puebla; la parte inferior con restos vegetales corresponde a los depósitos continentales del Jurásico inferior y medio, y la saperior a la serie marina del Jurásico superior. Por la tanto, según lo conocido hasta ahora, el Jurásico de Chiapas se divide litológica y estratigráficamente como el Jurásico de Oaxaca y de Puebla.

Arriba del Neocomiano existe todo el Cretácico, sobrepuesto por el Terciario. Esta última formación es arenosa, contiene corales y foraminiferos, pero nada de paqujodontos, por' lo que probablemente se trata de las capas basales del Terciario sobrepuestas concordantemente al Cretacico. Entre el supuesto Temiario y el Jurásico superior hay algo más de 1,500 metros de estratos cretácicos que se dividen litológica y estratigráficamente en tres partes: La serie inferior está formada por 150 metros de areniscas y margas, pizarras, caliza margosa y de areniscas y, pizarras rojas; en su parte basal contiene la fauna del Neocomiano, y'a 30 metros arriba de éste hay un banco de arenisca verdusca. con muo chos restos vegetales. Esta serie pertenece âl Cretácíco inferjor, sin que sea posjble actualmente fijar el límite estratigráfico superior exacto. Está sobrepuesta por cerca de cuatroeientos metros de caliza, conglomerado calizo y caliza con pedernal que contienen algunos invertebrados marinos, a saber Milíolidas y gasterópodos mal conservados, y que siśn debajo del Cretácico superior. por lo cual pertenecen al Cre. tácico medio.

Falta todavía el Cretácico -superior para completar la estratigrafía del ya mencionado Cretácico. Buese no reconoció la existencia de esta subdivisión, pero Sapper si la descubrió. y éste tenía razón, pues hay 1000 metros de capas de areniscas, margas, calizas margosas y arcillosas, y calizas que contienen muchos invertebrados marinos fósiles; en primer lugar paquiodontos, así como también foraminíferos, lamelibránquios, 
gasterópodos, corales, amonitas y algas calcáreas, de los cuarles son de importancia estratigráfica parte de los paquiodOlltos y las amonitas, que corresponden a especies ya conocidas del Viejo Mundo. Elstos son los fósiles de suma importancia estratigráfica que he recolectado en mis ocho exploraciones en Chiapas, y que permiten reconocer las subdivisiones del Cretácico superior. Las amonitas fueron determinadas por el Dr. C. Burckhardt, por lo que le estoy muy agradecido a mi amigo que, falleció desgraciadamente poco tiempo después de haber clasificado el material de Chiapas.

Del Cretácico superior, el Turoniano inferior no queda demostrado por la falta de fósiles caracteristicos. El Turoniano superior está C8'racterizado por la Distefanalla lombricaJis (d'Orb.) Dowrillé, Sauvagesia cí. da Rio Catullo, y el Radiolites alf. Iusitanicus Bayle, especies conocidas del Turoniano superior del Viejo Mundo, y por la Apricardia sp., que se encuentra en las capas con Hjppurites resectus varo mexicana de Apaxco, Méx., del Turoniano superior.

Es de mencionarse qUe en esta serie se encuentra la Sauvagesia degolyeri Stanton, que procede de su localidad eriginal, la Huasteca, de la parte inferior de las capes de San Felipe y la famosa Barrettia, conocida en Jamaica, Cuba y Guatemala, reconocida hasta ahora comp perteneciente al Senoniano superior. En realidad la Barrettia, pertenece al Turoniano superior, como lo he demostrado en un artícuJo reciente (8). La Barrettia viene acompañada por el Plagioptychus Toucasi Math. (5), ,otro paquiodonto del Turoniano de Europa.

En el límite de este piso y del siguiente, el Coniacjano, hay dos niveles de amonitas: el nivel inferior contiene Lytoceras (Gaudryceras) sp. indet., Pachydiscus peramplus varo Beyrensis Choffat, Acanthoceras sp. indet., y pertenece todavía al Turoniallo; el nivel superior contiene Hauericeras sp. jndet., género que caracteriza al Senoníano. Entre los dos niveles pasa,entonoes, el límite del Turoniano y del Senomano. 
El piso inferior de éste, el Coniaciano, contiene la Chiapa. sena radiolitiformis (Treehmann) Müllerried (4), la Pseudobarretia que son acompañadas por el Biradolites eanalicula. tus d'Orb., característico del Coniaciano de Francia, según Toncas; y de la Durania ef. austinensis, CuYo original procede del Coniaciano de Texas. El -Coniaciano como el Santoniano inferior de Chiapas están caracterizados por el Radiolites cf. subradiosus Toucas, y el Santoniano inferior, por el Vacei. nites giganteus d'Hombres-Firmas varo major Toncas, y el Radiolites cf. galloprovincialis Mathéron.

Por último queda comprobada la presencia del Campaniano por el Praeradiolites aft. subcoquandi Toucas, cuyo original procede, según dicho autor, de este piso. Poco más arriba de esta zoma, se encuentran en Ohiapas capas muy semejantes a las de Cárdenas, S. L. P. porque tienen Coralliochama, Biradiolites Aguilerae Boese, Aetaeonella occidentalis Boese y Biradiolites cardenasensis Boese. Esta última especie es muy semejante al Biradiolites Orbignyi Toucas del Campaniano de Francia.

Por esto y como hay paquiodontos más arriba de la serie en cuestión, me inclino a creer que las capas con Coralliochama (División de Cárdenas) pertenecen al Campaniano. De ningún modo pertenecen al 8en-oniano inferior como 10 indicó Boese. porque sobreponen al Santoniano en Chiapas. Al Campaniano y Maestrichtiano pertenecen entonces las capas con Coralliochama y las sobrepuesta,s, que contienen pocos paquiodontos; los estratos todavía más arriba, pero sin paquiodontos, son terciarios muy.probablemente.y ya de ninguna manera cretácicos.

El Cretácico superior de Chiapas contiene otros fósiles nlarinos aún, principalmente paquiodontos, conocidos casi exclusivamente de Jamaica y Cuba como se ve con claridad al comparar el material que yo traje de Obiapas', con los trabajos realizados por R. P. Whitfield, C. T. Trechmann, M. Sánchez Roig, H. DouviHé y R. H. Palmero Pertenece a una facies que existe en otras partes de la ,República Mexicana, pero que es muy poco conocida todavía, puesto que se han 
estudiado anteriormente las amonitas. Para terminar quiero mencionar que, las tres divisiones del Cretácico de Chiapas litológica y. estratigráficamente, corresponden a lo conocido ya en el oriente y sur de México, o sea al Cretácico inferior compuesto de rocas arenosas y margosas; al Cretácico medio de calizas, y alCretáeico superior de estratos variados.

\section{Resumen}

El complejo basal de la serie estratigráfica preterciaria de Chiapas, se compone de rocas metamorfizadas a intrusivas en gran parte anteriores al Paleozoico, lo que queda demostrado por el estudio ya hecho de la continuación del complejo basal en cuestión en el S. O. de los' E; . U. A.

Los estratos sobrepuestos al complejo basal son del Paleozoico superior, del Jurásico y del Cretácico. El Paleozoico superior está caracterizado en parte por fósiles ca,racterístioos del Carbonífero superior y del Pérmico. Bajo el Cretácico yace concordantemente el Jurásico, que contiene restos vegetales en la: parte inferior y algunos invertebrados marinos en la superior, representando respectivamente al Jurásico inferior y medio y al Jurásjico superior, bien conocidos de la mis-, ma facies en el sur del país. El Cretácico se divide en: Cretácico inferior, medio y superior y los fósiles de guía demuestran la presencia del Neocomiano, Turoniano superior, Coniaciano, Santoniano y Campaniano, estando presente, sin embargo, toda la serie cretácica que consiste de algo más de 1,500 metros de calizas, areniscas y margas sobrepuestas aparentemente por estratos del Terciario. 


\section{LISTA DE LITERATURA CITADA, REFERENTE A LA ESTRATIGRAFIA PRETERCIARIA DE CHIAPAS}

1.-José G. Aguilera, E. Ordón̄ez y R. J. Buelna; Bosquejo Geológico de México.-mInst. Geol. México, Bọl 4-6 p. 198, 1896.

'.-E. Boese: Reseña acerca de la gealogia de Chiapas y Tabasco. Inșt. Geol. México, Bol 20, 116 pp. 9 lámıs. 1905.

3._F. K. G. Mïllerried: Informe preliminar.-Chiapas.-1927, 1928 y 1929._Inst. Geol. Mérico, Foll. 36, 1\& ,pp. 1930.

4._F. K. G. Müllenied: Chiapasella. an paquiodonto extrañisimo de la Annérica.-Ansles Inst. Biol., t. 2, p. 243, 1931.

5.-F. K. G. Müllerried: El género Plagioptychus en México.-Ana. les Inst. Bicl. t. 4, p. 3, 1933.

5._F. K. G. Múllerried: Die Beziehungen del' Pachyodonten Amen. kas zu denen del' Alten Welt-Geol. Rundschar, Bd. 23a. p. 267, 1933.

G a) F. K. G. MüIlerried: Nota sobre el hallazgo de paquiodontos gi. gantescos en el Cretácico de Chiapas.-Anales Inst BioL t. V, No. 1. 1934, pp. $121 \cdot 122$.

7.-F. K. G. Müllerried: Geología Petrolera del Estado de Chiapas. -Inst. Geol. Méxjco, 1935. (Manuscrito).

8.-F. K. G. Müllerried: La edad estratigráfica de la Barrettia y formas cereanas.-Anales Inst, Biol 1.7, 1936.

'.-e. Sapper: Informe sobre la geografia física y la geología de los Estados de Chiapas y Tabasco,-Bol. Secr. Fom., 111, p. 187, 1894.

1O.-C. Sapper: Informe sobre la geografía física y la geología de la Península de Yucatán-Inst. Geol. México, Bol. 3, 58 pp. 6 láms. 1896.

11.-C. Sapper:' Uber Gebirgsbau und Boden des nordliehen Mittel amerika.--Peterm. Mitt., Erg., heft. 127, pp. VI 119, 3 cartas y 25 perfiles, 1899.

12.-W. A. Verwiebe: Geology of southern México oil fields.-Pan Am. Geol., Vol. 44 p. 121, 1925.

".-L. Waibel: Die Sierra Madre de Chiapas.-Mith Geograph. Ges. Hamburg, t. 43. p. 12. 1933.

14._B. Willis: Index to the stratigraphy of North America. - U. S. Geol. Survey, frof. Paper 71, p. 425, 1912. 